\title{
Paroxysmal Dyskinesias in the Lethargic Mouse Mutant
}

\author{
Zubair Khan and H. A. Jinnah \\ Department of Neurology, Johns Hopkins Hospital, Baltimore, Maryland 21287
}

Lethargic mutant mice carry a mutation in the CCHB4 gene, which encodes the $\beta 4$ subunit of voltage-regulated calcium channels. These mutants have been shown to display a complex neurobehavioral phenotype that includes EEG discharges suggestive of absence epilepsy, chronic ataxia, and hypoactivity. The current studies demonstrate a fourth element of their phenotype, consisting of transient attacks of severe dyskinetic motor behavior. These attacks can be triggered by specific environmental and chemical influences, particularly those that stimulate locomotor activity. Behavioral and EEG analyses indicate that the attacks do not reflect motor epilepsy, but instead resemble a paroxysmal dyskinesia. The lethargic mutants provide additional evidence that calcium channelopathies can produce paroxysmal dyskinesias and provide a novel model for studying this unusual movement disorder.

Key words: lethargic; mouse mutant; calcium channel; channelopathy; paroxysmal dyskinesia; movement disorder; epilepsy
Ion channels play a critical role in both intercellular and intracellular communication in the nervous system. Multiple human neurological disorders have been linked with defects in calcium, sodium, potassium, or chloride channels (Greenberg, 1997; Barchi, 1998). For example, mutations in the pore-forming $\alpha 1 \mathrm{~A}$ subunit of P/Q-type voltage-gated calcium channels cause several different human diseases, including paroxysmal ataxia, chronic progressive ataxia, familial hemiplegic migraine, and absence epilepsy (Jen, 1999; Tournier-Lasserve, 1999; Jouvenceau et al., 2001).

Mutations that affect P/Q-type calcium channels have also been identified in several different mouse mutants, each displaying a distinct abnormal neurobehavioral syndrome. Four different mutations that affect the $\alpha 1 \mathrm{~A}$ subunit have been identified in tottering, leaner, rolling, and rocker mice (Fletcher et al., 1996; Doyle et al., 1997; Mori et al., 2000; Zwingmann et al., 2001). In addition, two $\alpha 1 \mathrm{~A}$ knock-out mice have been generated (Jun et al., 1999; Fletcher et al., 2001). The physiological properties of the $\alpha 1 \mathrm{~A}$ pore are influenced by three auxiliary subunits; mutations in each of these subunits have also been identified in strains of mutant mice with abnormal neurobehavioral syndromes (Burgess et al., 1997; Letts et al., 1998). These include lethargic mice with mutations of a $\beta 4$ subunit (Burgess et al., 1997), ducky mice with mutations of an $\alpha 2 \delta 2$ subunit (Barclay et al., 2001), and stargazer and waggler mice with mutations of a $\gamma$ subunit (Letts et al., 1998).

The mutation in the $\beta 4$ subunit of lethargic mice results in a complex neurobehavioral phenotype. These mutants have been studied extensively as a model for absence epilepsy because of the presence of brief staring spells in association with $2-4 \mathrm{~Hz}$ polyspike discharges in EEG recordings (Hosford et al., 1995a,b; Aizawa et al., 1997; Hosford and Wang, 1997). In addition, an unsteady and ataxic gait emerges $2-3$ weeks after birth and

\footnotetext{
Received March 8, 2002; revised May 10, 2002; accepted June 3, 2002.

This work was supported by National Institutes of Health Grants NS01985 and NS40470. We thank Ellen J. Hess for reviewing this manuscript.

Correspondence should be addressed to Dr. H. A. Jinnah, Meyer Room 6-181, Department of Neurology, Johns Hopkins Hospital, Baltimore, MD 21287. E-mail: hjinnah@jhmi.edu

Copyright (C) 2002 Society for Neuroscience $\quad 0270-6474 / 02 / 228193-08 \$ 15.00 / 0$
}

persists throughout adulthood. Unlike other ataxic mice, characteristically slow and hesitant motor behavior also develops in lethargic mutants, a phenomenon for which the name lethargic was originally coined (Dickie, 1964). Early reports also anecdotally noted the occurrence of transient attacks of more severe motor disability, with falling and persistent abnormal postures (Dickie, 1964; Dung and Swigart, 1971), although the nature of these attacks has never been fully characterized.

In the current studies, the motor syndrome of the lethargic mutants was examined in detail and shown to consist of a baseline of ataxia with intermittent attacks that are reliably triggered by specific environmental or pharmacological influences. These attacks can be clearly dissociated from the EEG abnormalities and do not represent motor epilepsy. Instead, the attacks appear to represent a form of paroxysmal dyskinesia.

\section{MATERIALS AND METHODS}

Animals. Homozygous lethargic females $\left(\mathrm{cachb} 4^{\text {lh/lh }}\right)$ and normal congenic $(\mathrm{C} 3 \mathrm{H} \times \mathrm{C} 57 \mathrm{BL} / 6 \mathrm{~J})$ males were obtained from The Jackson Laboratory (Bar Harbor, ME) and bred to generate heterozygotes. The heterozygotes were then crossed to generate homozygous lethargic mice along with normal and heterozygous littermates as controls. The mice were weaned at 4 weeks of age and housed in groups of two to eight with a 14/10 hr light/dark cycle and access to food and water ad libitum. Behavioral testing was conducted during the light phase unless otherwise specified. Animals were 12-16 weeks of age at the time of behavioral testing, unless otherwise noted. All animal procedures were conducted in accordance with institutional and National Institutes of Health guidelines for the treatment of experimental animals.

Rotarod. Coordinated motor skills were assessed via the rotarod test (Columbus Instruments, Columbus, $\mathrm{OH}$ ). Animals were placed on top of a 4 -cm-diameter rod, which began to rotate within $1 \mathrm{sec}$. The rotation speed increased from 4 to $20 \mathrm{rpm}$ over a $5 \mathrm{~min}$ period; the average time to falling was determined from three trials for each mouse.

Cling test. Climbing and hanging skills were assessed via the cling test. A $20 \times 20 \mathrm{~cm}$ platform with a $6 \mathrm{~mm}$ grid of wire mesh surrounded by a $5 \mathrm{~cm}$ frame was suspended $50 \mathrm{~cm}$ above a soft landing area, so that the mice could not escape the platform except by falling. Mice were placed on top of the frame and allowed to habituate for $60 \mathrm{sec}$, after which the grid was rotated $90^{\circ}$ and held in the vertical position for $60 \mathrm{sec}$, and then rotated another $90^{\circ}$ and held in the inverted position for $60 \mathrm{sec}$. The average time to falling was determined from three trials for each mouse.

Gross motor activity. Photocell activity chambers were used to quantify the gross motor activity of the mutants. The chambers consisted of $20 \times$ $40 \mathrm{~cm}$ Plexiglas boxes with four infrared beams spanning the short axis 
and eight infrared beams spanning the long axis (San Diego Instruments, San Diego, CA). Unless otherwise noted, mice were placed singly in the chambers at 9 P.M. at the onset of the normally active nocturnal period. Beam breaks were recorded automatically by computer every $10 \mathrm{~min}$, with no habituation period, for $10 \mathrm{hr}$.

When the testing paradigm precluded the use of the photocell chambers, gross motor activity was estimated using a semiquantitative score according to the level of activity: 0, resting with eyes closed; 1 , awake with minimal motor behavior; 2, normal spontaneous activity; and 3 , hyperactive motor behavior.

Quantification of attacks. A time-sampling behavioral inventory method (Sahgal, 1993) was used to quantify dyskinesias during attacks. Each animal was observed for exactly $60 \mathrm{sec}$ at $10 \mathrm{~min}$ intervals for $1 \mathrm{hr}$. For each interval, specific target behaviors identified from preliminary observations of attacks were recorded: circling (at least one full $360^{\circ}$ turn), listing (leaning to one side), abnormal truncal postures (exaggerated flexion or flattening), facial twitching (perioral or periocular), clonic forelimb or hindlimb movements (three or more rapid limb jerks), and tonic forelimb or hindlimb movements (sustained abnormal postures $\geq 1$ sec in duration). Scoring was conducted with the observer blinded to treatment whenever possible. Composite dyskinesia scores for normal animals were always $<2$; the maximum potential score was 36 .

Several environmental factors were methodically evaluated to determine their influence on the severity of the attacks. Two procedures were used to investigate the influence of motor activity. First, mice were scored after they were transferred to a small $(10 \times 10 \mathrm{~cm})$, medium $(17 \times$ $28 \mathrm{~cm})$, or large $(24 \times 42 \mathrm{~cm})$ Plexiglas cage. Next, the mice were scored undisturbed in their $24 \times 42 \mathrm{~cm}$ home cages during daytime (sleeping period), early nocturnal (most active), and late nocturnal (awake, less active) hours. The influence of stress was also examined under two different conditions. First, mice were scored while subjected to a $0.1 \mathrm{~mA}$ electric shock for $1 \mathrm{sec}$ every $10 \mathrm{~min}$ for $1 \mathrm{hr}$ in a shuttle box. Next, mice were restrained inside $50 \mathrm{cc}$ plastic syringes for $10 \mathrm{~min}$ as described previously (Campbell and Hess, 1999) and then scored for $1 \mathrm{hr}$ after they were released into their home cages. The influence of vestibular stimulation was assessed by scoring animals in their home cages after they were subjected to $10 \mathrm{~min}$ of gentle vibration or to an orbital shaker rotating at $15 \mathrm{rpm}$ (LabLine, Dubuque, IA).

The influence of several drugs on the severity of attacks was also investigated. For drugs determined from preliminary studies to promote attacks, mice were scored in their home cages $3 \mathrm{hr}$ after habituating from transportation to the lab. Under these conditions, low baseline dyskinesia scores limited potential ceiling effects. For drugs that attenuated attacks, mice were scored after being placed in a large cage immediately after transportation to the lab. Under these conditions, high baseline scores limited potential floor effects.

Treadmill. A treadmill (Columbus Instruments) was used to examine the temporal profile of dyskinesias. Mice were habituated to the treadmill on four to five separate occasions and trained to walk at a moderate pace of $8.5 \mathrm{~m} / \mathrm{min}$ to avoid a $0.1 \mathrm{~mA}$ electric shock. On the test day, they were habituated to the apparatus for $2 \mathrm{hr}$, with the treadmill and electric shocking device turned off. The treadmill was then turned on, and the mice walked continuously until the onset of dyskinesias. Once the dyskinesias became sufficiently severe enough that they could no longer walk, the treadmill was turned off. Dyskinesia scores were recorded at 1 min intervals for $20 \mathrm{~min}$ beginning from the time the treadmill was started.

Electrophysiology. For EEG recordings, mice were anesthetized with $20 \mathrm{ml} / \mathrm{kg} 2 \%$ 2,2,2-tribromoethanol in saline (Fluka, Neu-Ulm, Germany) with supplemental gaseous methoxyflurane as needed (ScheringPlough Research Institute, Union, NJ). After shaving the hair and cleansing the surgical sites with $2 \%$ benzylalkonium (Sigma, St. Louis, MO) and ethanol, a $1 \mathrm{~cm}$ incision was made in the lower abdomen. A TA10EAF20 telemetry transmitter (Data Sciences International, St. Paul, MN) was inserted into the peritoneal space, and the attached stainless steel wires insulated with silicone were tunneled subcutaneously around the abdomen to the base of the skull. A second $1 \mathrm{~cm}$ incision was made on the scalp and two miniature MX-00120-1F screws (Small Parts Inc., Logansport, IN) were attached to the skull over the cortex, $\sim 2 \mathrm{~mm}$ anterior to the bregma and $2 \mathrm{~mm}$ to either side of the sagittal suture. The end of each electrode wire was exposed, looped around a screw with a small amount of \#16034 colloidal liquid silver to promote electrical contact (Ted Pella Inc., Redding, CA), and secured with Loctite \#411 instant adhesive (Loctite, Rocky Hill, NC). Both wounds were closed with Nexaband formulated cyanoacrylate tissue adhesive (Veterinary
Products Laboratories, Phoenix, AZ), secured with 4-0 silk sutures, and coated with Tritop antiseptic and anesthetic ointment (Pharmacia \& Upjohn Co., Clayton, NC). The animals were then placed on a heating pad set to $30^{\circ} \mathrm{C}$ and allowed to recover. They received $50 \mathrm{mg} / \mathrm{kg}$ chloramphenicol twice daily for $3 \mathrm{~d}$ to reduce the risk of infection.

A postoperative recovery period of at least 3 weeks was allowed before making recordings. Digital EEG recordings were collected with an RPC-1 telemetry receiver (Data Sciences International) at a sampling rate of $1000 \mathrm{~Hz}$ and filter cutoff of $70 \mathrm{~Hz}$. Traces were analyzed with Dataquest 2.1 software (Data Sciences International). Polyspike discharges were identified as consecutive trains of at least three sharp waves with an amplitude of at least twice the background amplitude, a frequency of 2-4 Hz, and a duration of $<50$ msec.

Data analysis. Two-way ANOVA with genotype and age as the main variables was used to compare the longitudinal data collected from normal and lethargic mice at different ages for the rotarod test, the cling test, and the photocell recordings. To account for the application of five different tests to the same cohort of animals, the Bonferroni method was used to define a $p$ value of $<0.01$ as the requirement for statistical significance. Two-way ANOVA with genotype and time as the main variables was used to compare the simultaneous photocell recordings and dyskinesia scores. One-way ANOVA was used to analyze the dyskinesia scores from the longitudinal study, the drug dose-response studies, and the EEG study. Post hoc Tukey $t$ tests were used when appropriate.

\section{RESULTS}

\section{Longitudinal motor performance}

To address the possibility of changes in the motor syndrome with age, three standard tests of motor function were applied to a cohort of lethargic and normal mice at specific intervals from 4 to 40 weeks of age. Lethargic mutants performed extremely poorly on the rotarod, in comparison with normal mice, which stayed on the rod for nearly the maximum allowed time (Fig. $1 A$ ). Lethargic mutants also did poorly on the cling test in comparison with normal mice (Fig. $1 B$ ). The performance of both normal and lethargic mice declined with age for both tests.

Despite their poor performance on the rotarod and cling tests, lethargic mutants displayed gross levels of motor activity similar to those of normal mice during overnight recordings in the photocell chambers (Fig. 1C). This result was unexpected, in view of the characteristically slow and hesitant motor behavior of these mutants. Analysis of the temporal patterns of activity in the photocell chambers revealed reduced levels of activity only during the initial period of the recording (Fig. 1D). This result suggested that the apparently slow and hesitant motor behavior might be a transient phenomenon limited to the initial test period.

\section{Identification of transient dyskinesias}

To determine the basis for reduced motor activity during the initial test period in the photocell chambers, detailed open-field observations of $>40$ lethargic mutants were made. At baseline they demonstrated features typical of ataxia: a wide stance, imprecise limb placement, poor timing and interlimb coordination, and a staggering gait. Falls were rare, and functional disability was minor. Superimposed on these relatively minor abnormalities were transient periods of more severe motor disability. These attacks were most prominent when the mutants were placed in a new testing environment and abated in 60 min, coinciding with the transient period of reduced locomotor activity in the photocell chambers (Fig. 2). In comparison, normal mice did not display any of the abnormal movements observed in lethargic mice (Fig. 2).

The attacks consisted of a collection of dyskinetic behaviors, including circling, listing to one side, exaggerated flexion or extension of the trunk, falling (with inability to regain the upright posture), twitching of the perioral or periocular muscles, clonic 

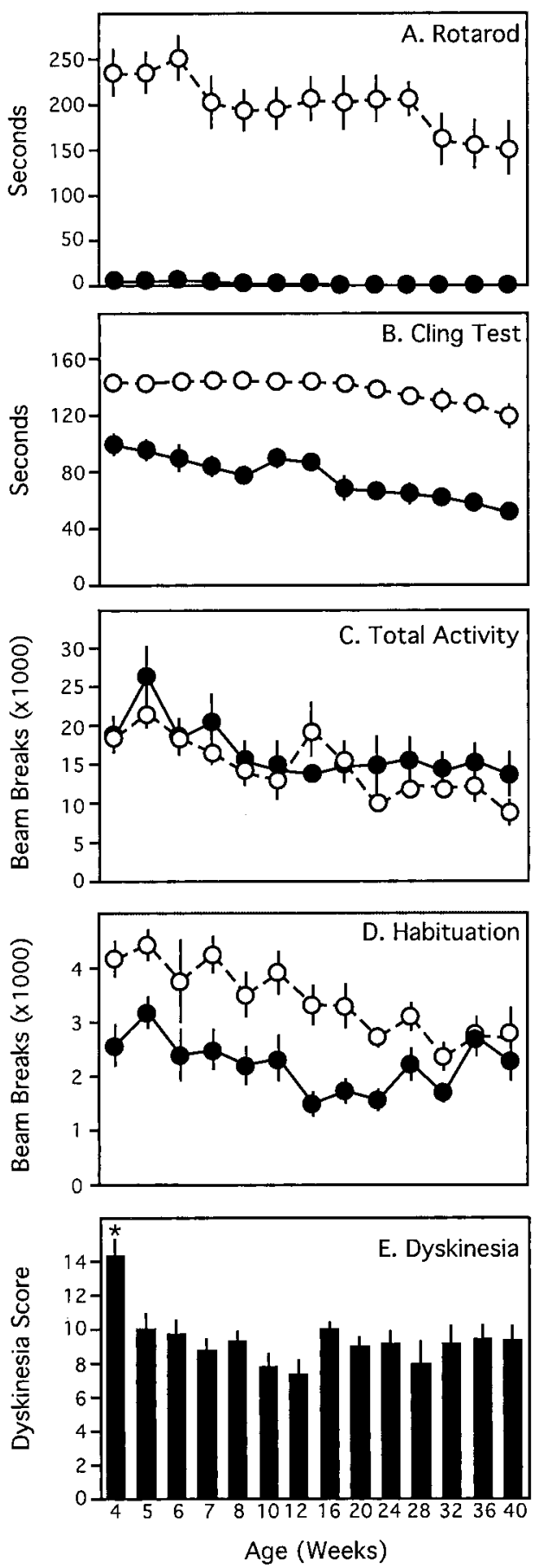

Figure 1. Age-related changes in motor function. $A$, Rotarod. B, Cling test. $C$, Gross motor activity during overnight recordings. $D$, Gross motor activity during the first hour of testing. $E$, Dyskinesia scores. Results reflect average values \pm SEM for 10-12 normal mice (open circles) and 8-10 lethargic mutants ( filled circles) at each time point. Two-way ANOVA for the rotarod revealed significant effects for genotype $\left(F_{(1,261)}=1308 ; p<\right.$ $0.001)$ and age $\left(F_{(12,261)}=2.5 ; p<0.005\right)$. For the cling test, there were also significant effects for both genotype $\left(F_{(1,261)}=1435 ; p<0.001\right)$ and age $\left(F_{(1,261)}=14.0 ; p<0.001\right)$. For overnight activity, there was a significant effect of age $\left(F_{12,261}=4.4 ; p<0.001\right)$ but no significant effect for genotype $\left(F_{(1,261)}=4.5 ; p=0.04\right)$. For the first hour of activity, there were again significant effects for genotype $\left(F_{(1,261)}=65.3 ; p<0.001\right)$ and age $\left(F_{(12,261)}=3.8 ; p<0.001\right)$. For dyskinesia scores, one-way ANOVA revealed significant differences among ages $\left(F_{(13.149)}=2.8 ; p<0.002\right)$; post hoc Tukey $t$ tests indicated that the 4 -week-old group was significantly different (asterisk) from all other age groups $(p<0.01)$.

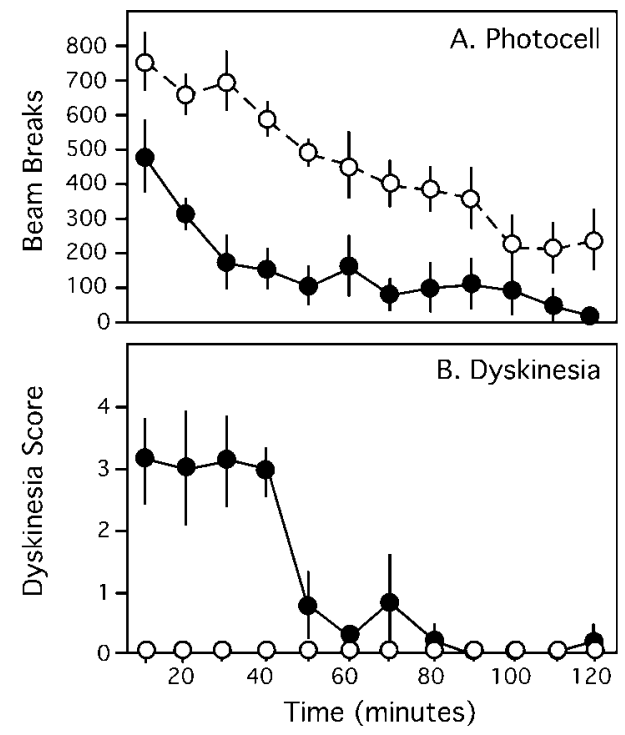

Figure 2. Temporal profile of gross motor activity $(A)$ and dyskinesia scores $(B)$. Results reflect average values \pm SEM for six normal mice (open circles) and seven lethargic mutants (filled circles) that were 12-16 weeks of age. Two-way ANOVA revealed significant differences between genotypes $\left(F_{(1,132)}=222.8 ; p<0.001\right)$ and time $\left(F_{(11,132)}=18.3 ; p<\right.$ $0.001)$ for gross motor activity. A similar analysis also showed significant differences between genotype $\left(F_{(1,132)}=123.5 ; p<0.001\right)$ and time $\left(F_{(11,132)}=13.2 ; p<0.001\right)$ for dyskinesia scores.

movements of the limbs, or tonic extension or retraction of the limbs (Fig. 3). These attacks were most prominent at 4 weeks of age (Fig. 1E). By 5-6 weeks of age, some of the behaviors became less prominent (e.g., circling, listing, falling, and facial twitching), whereas others persisted (e.g., clonic or tonic movements of the limbs or trunk). The features and overall severity of attacks then remained relatively stable up to 40 weeks of age (Fig. $1 E$ ). There was no difference between the dyskinesia scores of male and female mutants.

\section{Environmental influences on dyskinesia attacks}

To determine the factors responsible for triggering attacks, the mutants were exposed to different environmental conditions. To determine whether placing the animals in a novel test cage was responsible for initiating attacks, mutants were scored after they were picked up and transferred into a cage similar to the home cage or transferred into a smaller or larger cage. All cages promoted alertness because of their novelty; but the small cage permitted only limited ambulation, whereas the large cage encouraged exploratory ambulation. The lowest dyskinesia scores were observed in the small cages and the highest scores were observed in the large cages (Fig. 4A). Because this result suggested that motor activity rather than cage novelty might be responsible for triggering attacks, dyskinesia scores were compared when the animals were observed undisturbed in their home cages during the light period (normal inactive period), at the onset of the dark period (beginning of active period), and 6-8 hr after the onset of the dark period (end of active period). The lowest scores were observed during the inactive period, the highest scores were observed at the onset of the active period, and intermediate scores were observed at the end of the active period (Fig. $4 B$ ). This result confirmed that motor activity, and not the stress of a novel environment, was influencing the dyskinesia scores.

The influence of stress was tested by scoring the mice while 

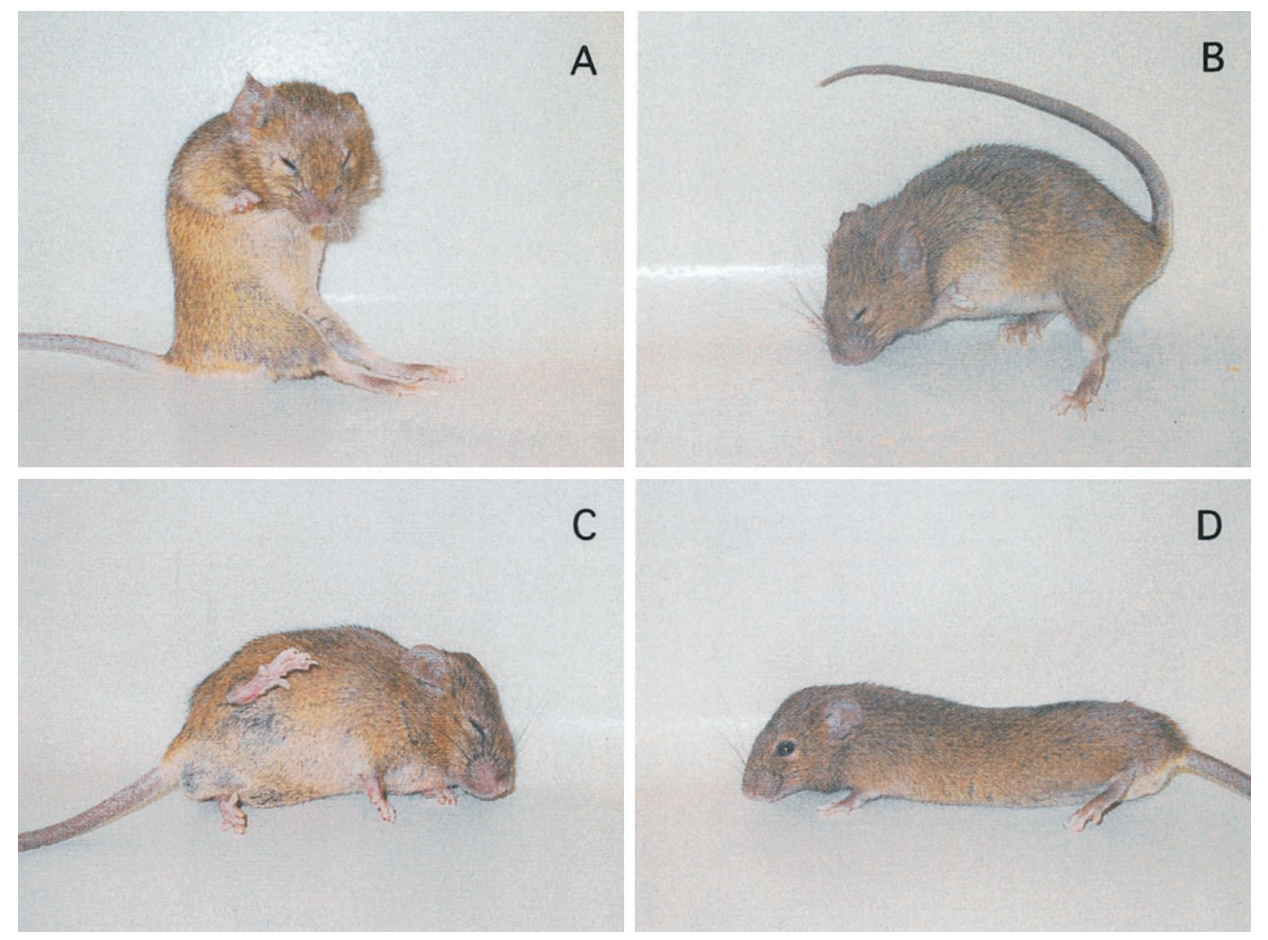

Figure 3. Dyskinetic movements during attacks in lethargic mutants. $A$, Exaggerated truncal flexion with forepaw clonus. $B$, Truncal flexion with tonic forelimb retraction. $C$, Listing left with tonic rear limb retraction. $D$, Exaggerated truncal flattening.

they were subjected to intermittent electric shock or after they were restrained inside a $50 \mathrm{cc}$ syringe for $10 \mathrm{~min}$. Both procedures caused a significant increase in dyskinesia scores (Fig. 4C). However, neither procedure could exclude the influence of motor activity. Intermittent electric shock was associated with jumping in response to the shock; restraint stress was typically associated with a brief phase of hyperlocomotion immediately after the animal was freed from the syringe. Dyskinesias were not in-

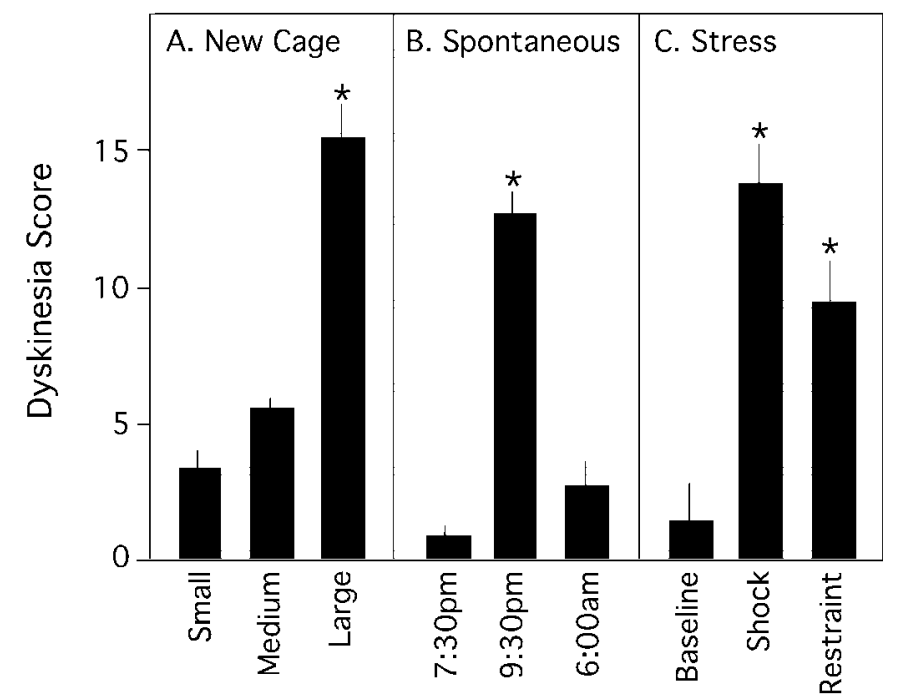

Figure 4. Environmental influences on total dyskinesia scores in lethargic mice. Results reflect average values \pm SEM for 12-13 mice at 12-16 weeks of age for each condition. $A$, Cage size. $B$, Time of day. $C$, Stress. ANOVA revealed significant differences among the conditions $\left(F_{(8,103)}=\right.$ 31.5; $p<0.001$ ). Post hoc Tukey tests provided $p<0.001$ (asterisks) for each of the following comparisons: large cage scores were significantly higher than both small and medium cage scores; 9:30 P.M. scores were significantly higher than both the 7:30 P.M. and 6:00 A.M. scores; and shock or restraint stress scores were significantly higher than baseline scores. creased by vestibular stimulation on an orbital shaker and were increased slightly by gentle vibration (data not shown).

\section{Temporal profile of dyskinesia attacks}

Although average dyskinesia scores suggested an attack duration of 40-60 min (Fig. 2), individual mutants were observed to have multiple shorter attacks during this period. To obtain a more precise estimate of the duration of attacks and to confirm the role of motor activity, six young adults (12-16 weeks of age) were individually recorded while walking on a treadmill. Dyskinetic behavior was absent when the treadmill was stationary, but be-

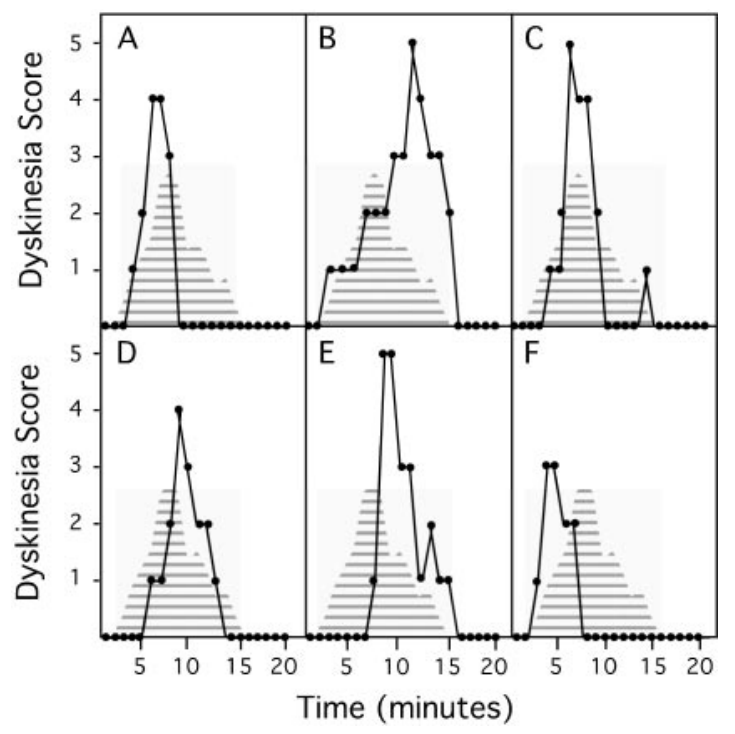

Figure 5. Temporal profile of dyskinesias in lethargic mice. Mice at 12-16 weeks of age were habituated for $2 \mathrm{hr}$ to the stationary treadmill and then scored every $60 \mathrm{sec}$ for $20 \mathrm{~min}$ after starting it in motion. The treadmill was stopped at the onset of an attack. Filled circles show data for individual mice; the striped zone depicts average values for the entire group. 


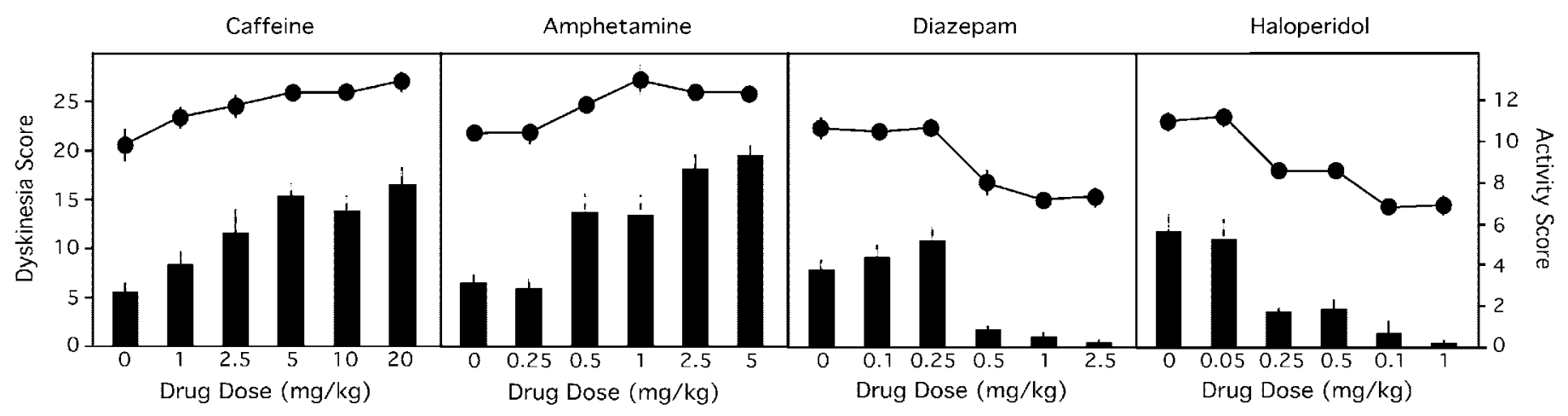

Figure 6. Relationship between gross level of activity (line graphs) and total dyskinesia scores (bar graphs) 10-15 min after administration of caffeine, amphetamine, diazepam, or haloperidol. Results reflect average values \pm SEM for six to eight mice at $12-16$ weeks of age per condition. ANOVA for activity scores revealed significant effects for each drug: caffeine $\left(F_{(5,30)}=5.7 ; p<0.001\right)$, amphetamine $\left(F_{(5,30)}=6.3 ; p<0.001\right)$, diazepam $\left(F_{(5,36)}=\right.$ 16.5; $p<0.001)$, and haloperidol $\left(F_{(5,36)}=25.6 ; p<0.001\right)$. ANOVA for dyskinesia scores also revealed significant effects for each drug: caffeine $\left(F_{(5,30)}=8.0 ; p<0.001\right)$, amphetamine $\left(F_{(5,30)}=17.3 ; p<0.001\right)$, diazepam $\left(F_{(5,36)}=28.8 ; p<0.001\right)$, and haloperidol $\left(F_{(5,36)}=17.0 ; p<0.001\right)$. Across all drugs, the overall Spearman rank correlation between activity level and dyskinesia score was 0.87 .

came severe enough that the treadmill had to be stopped an average of $3.7 \pm 0.7 \mathrm{~min}$ after it was started (Fig. 5). The dyskinesia attacks lasted an average of $7.8 \pm 1.5 \mathrm{~min}$ before returning to baseline. There was no obvious refractory period after an attack, because dyskinetic behavior could be induced within minutes after turning the treadmill on again (data not shown).

In view of observations suggesting that young mice had more severe attacks than older mice, a group of older adults (12-14 months of age) were also recorded on the treadmill. In this group, dyskinetic behavior severe enough to prevent ambulation occurred in only four of six mice. Minor dyskinesias developed in two mice, but the animals were capable of continued ambulation. The average time to the onset of dyskinesias in the remaining four mice was $12.0 \pm 4.0 \mathrm{~min}$, with an average duration of $4.2 \pm 0.7$ min. These results confirm previous suggestions that attacks are less prominent in older mice.

\section{Chemical influences on dyskinesia attacks}

Overall, the highest dyskinesia scores occurred in association with environmental conditions that promoted increased levels of motor activity. To determine whether the pharmacological stimulation of motor activity would result in higher dyskinesia scores, groups of six to eight mutants were habituated to their test cages for 3-4 hr to provide a low baseline level of dyskinesia and then treated with one of two locomotor stimulants. Doses of amphetamine or caffeine that produced a mild stimulation of locomotion both provoked dose-dependent increases in dyskinesia scores in lethargic mice (Fig. 6).

To determine whether the pharmacological suppression of motor activity would result in lower dyskinesia scores, unhabituated animals with a high baseline level of dyskinesia were treated with one of two drugs that suppress motor activity. Doses of diazepam and haloperidol that produced moderate suppression of locomotion without inducing sleep both provoked a dose-dependent decrease in dyskinesia scores in lethargic mice (Fig. 6).

\section{Electrophysiology}

To determine whether the attacks were associated with epileptiform brain activity, dyskinesia scores and EEGs were recorded in lethargic mice pretreated with saline or $200 \mathrm{mg} / \mathrm{kg}$ ethosuximide. The EEG of saline-treated lethargic mice displayed frequent abnormal polyspike discharges (Fig. $7 A$ ), but there was no corre- lation between these discharges and any of the dyskinetic behaviors. Ethosuximide almost completely eliminated the polyspike discharges, while simultaneously increasing the severity of dyskinesias (Fig. $7 B, C$ ). A dose of $10 \mathrm{mg} / \mathrm{kg}$ nifedipine provoked the opposite phenomenon: polyspike discharges increased and dyskinesia scores decreased (Fig. $7 B, C$ ). Neither ethosuximide nor nifedipine improved the motor performance of lethargic mice on the rotarod or cling tests (data not shown).

\section{DISCUSSION}

\section{The motor syndrome of lethargic mutants}

The motor syndrome of the lethargic mutant mice consists of a baseline of mild ataxia with intermittent attacks of much more severely disabling motor dysfunction. These attacks occur in all lethargic mutants and consist of a collection of different abnormal movements. The attacks can be reliably provoked by a variety of environmental or chemical influences, particularly those that influence motor activity. The attacks are likely to be responsible at least in part for the very poor performance of these mutants on the rotarod, which requires active ambulation. In contrast, these mutants fare comparatively better on the cling test, which requires only hanging in one spot.

As suggested previously (Dickie, 1964; Dung and Swigart, 1971), the occurrence of motor attacks raises the possibility that the lethargic mutants have epileptic seizures. However, motor epilepsy is an inadequate explanation for these attacks, for several reasons. First, the varied morphology of the dyskinetic movements is quite uncharacteristic of the more commonly stereotyped tonic or clonic manifestations of motor epilepsy. Second, the duration of the attacks is uncharacteristically long for epileptic seizures, which typically last for less than $1 \mathrm{~min}$. Third, the expected depression of consciousness during and after an epileptic seizure is absent from the attacks displayed by lethargic mice. Fourth, repeated attacks can be observed without the usual postictal refractory period associated with motor epilepsy. Fifth, the attacks are reliably triggered by environmental influences that do not usually precipitate epileptic seizures. Although strong sensory phenomena or stress may provoke epileptic seizures in susceptible strains of mice, augmented motor activity usually does not (Todorova et al., 1999). Finally, EEG recordings reveal no features suggestive of epilepsy before, during, or after an attack. 


\section{A. EEG Baseline}

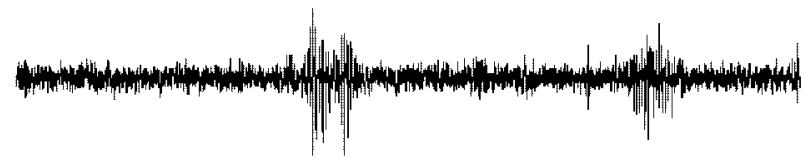

Ethosuximide
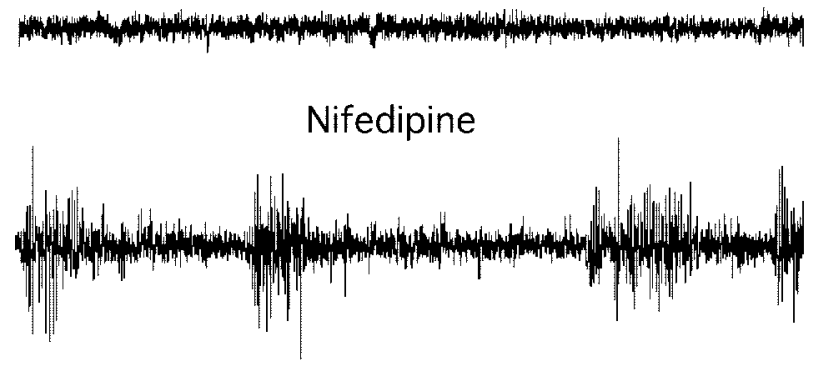
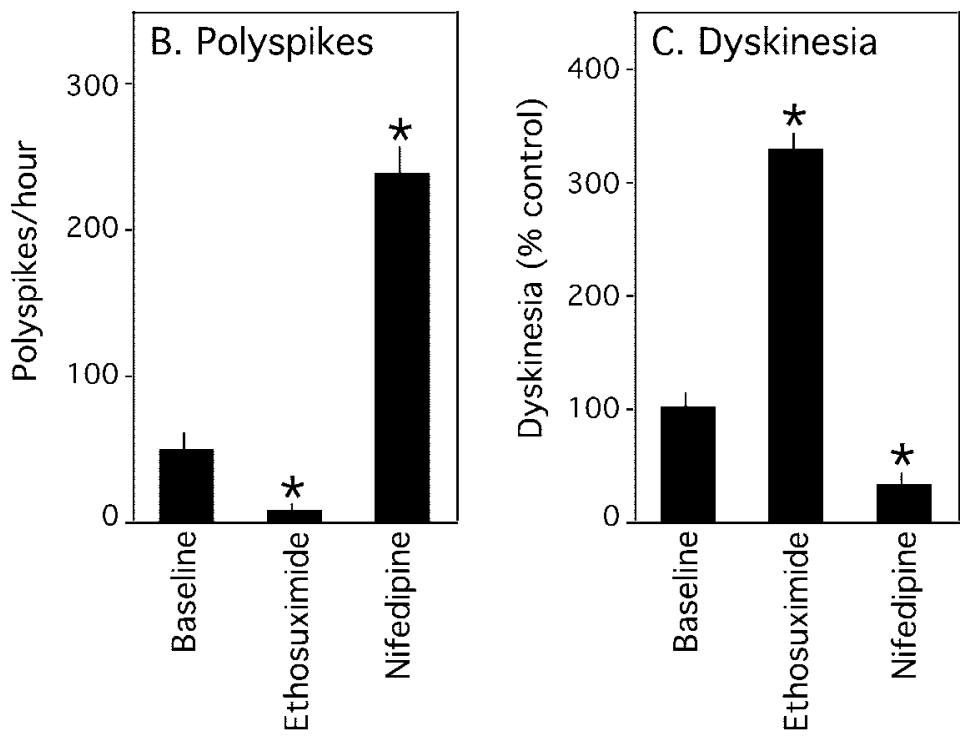

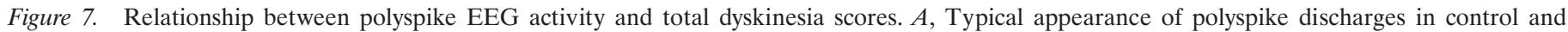

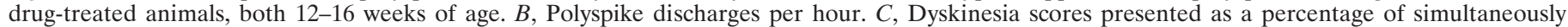

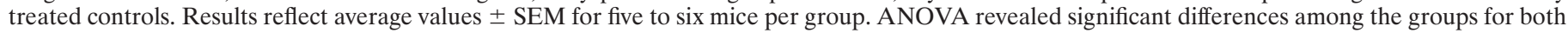

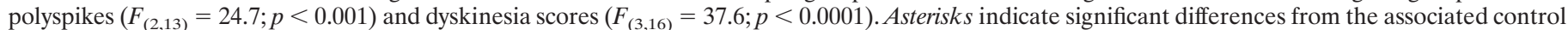
group $(p<0.001)$.

Instead, many features of the attacks exhibited by lethargic mice suggest that they represent a paroxysmal movement disorder.

\section{Paroxysmal movement disorders in humans}

The human paroxysmal movement disorders consist of a heterogeneous group of conditions; multiple attempts have been made to classify them into more meaningful subgroups (Kertesz, 1967; Lance, 1977; Fahn and Marsden, 1994; Demirkiran and Jankovic, 1995). Several features important for discriminating among different subtypes have been identified. The most important of these is whether the predominant abnormal movements are ataxic or dyskinetic in appearance. The paroxysmal ataxias are currently subdivided into episodic ataxia types 1 and 2, depending on associated features and attack duration.

The paroxysmal dyskinesias are subdivided into three groups. Dystonia is often the most common and severe manifestation of all three groups; but the less specific term dyskinesia is often used to acknowledge the existence of a variety of different motor signs, including chorea, athetosis, ballismus, tremor, or clonic jerks. An important feature useful for discriminating among the three subtypes of paroxysmal dyskinesias is what triggers attacks. The attacks of paroxysmal nonkinesigenic dyskinesia are often triggered by stress, caffeine, or alcohol. In comparison, attacks in paroxysmal kinesigenic dyskinesia are triggered predominantly by sudden movement; but they may also be triggered by stress, startle, muscle vibration, or passive limb movements. The attacks of paroxysmal exertional dyskinesia are triggered by prolonged exercise. The duration and frequency of attacks also help to discriminate among the paroxysmal dyskinesias. Attacks in paroxysmal nonkinesigenic dyskinesia are relatively prolonged (30 min to several hours) but infrequent (up to a few attacks per day). In contrast, attacks in paroxysmal kinesigenic dyskinesia are very short (a few seconds to a few minutes) but occur frequently (up to 100 per day). Those of paroxysmal exertional dyskinesia are intermediate in duration, with a frequency related to the level of physical exertion.

\section{Paroxysmal dyskinesias in lethargic mutants}

Although mutant mice cannot be expected to match precisely the diagnostic categories derived from related human conditions, the paroxysmal movement disorder of the lethargic mutants most resembles the paroxysmal kinesigenic dyskinesias. Lethargic mice display a baseline of mild ataxia, but their attacks do not resemble those of the paroxysmal ataxias. Instead, the attacks resemble a paroxysmal dyskinesia because they include a variety of dyskinetic behaviors without a predominant morphology (Fig. 3). The tonic extension and retraction of the limbs and abnormal truncal postures during attacks have a clearly dystonic quality, but the faster and more rapid limb jerks have a choreiform or clonic appearance. Although the dyskinesia attacks are promoted by motor activity, they do not require prolonged exercise, as in paroxysmal exertional dyskinesia. Instead, the attacks can be observed within minutes of very brief motor activity, may last for only a few minutes, and may occur many times per hour (Figs. 2, 6). All of these features are similar to those observed in the paroxysmal kinesigenic dyskinesias.

In view of the observation that movement precipitates dyskinesias, it is interesting to speculate that the characteristically slow and hesitant gait for which these mutants received the name lethargic may not be a direct result of impaired neuromotor systems but rather a behavioral strategy learned by the animals as a means to reduce the frequency of attacks. Lethargic mutants are capable of running quickly during the first few minutes on a treadmill, albeit with an ataxic gait. This observation indicates that they are physically capable of moving more quickly. The hypokinetic behavior of the lethargic mutants cannot be directly attributed to their ataxic gait, because hypokinesis is not typical of other ataxic mouse mutants with cerebellar dysfunction. In addition, the severe attacks of young lethargic mice attenuate over 1-3 months of age, coincident with the emergence of lethargic behavior. The suggestion that the slow and hesitant motor behavior of the lethargic mutants is a learned strategy is not unprece- 
dented, because human subjects with dyskinesias provoked by movement often use similar strategies to prevent or abort attacks (Kertesz, 1967; Sadamatsu et al., 1999; Munchau et al., 2000).

\section{Pathophysiology of paroxysmal movement disorders}

At the molecular level, several paroxysmal motor disorders have been linked with mutations in genes encoding ion channels. Among humans, episodic ataxias types 1 and 2 are associated with mutations that affect calcium or potassium channels (Bhatia et al., 2000). Several human disorders featuring paroxysmal paralysis have also been associated with mutations in calcium or sodium channels (Bhatia et al., 2000). In mice, defects in genes encoding or influencing the function of calcium channels are the basis for paroxysmal dyskinesias in tottering mice (Fletcher et al., 1996; Doyle et al., 1997; Fureman et al., 2002) and ducky mice (Barclay et al., 2001).

Lethargic mutants carry a four nucleotide insertion into a splice donor consensus site of the $\mathrm{CCHB} 4$ gene, resulting in exon skipping and translational frameshift (Burgess et al., 1997; Lin et al., 1999). The mutation is predicted to produce a truncated $\beta 4$ protein that is missing $\sim 60 \%$ of its $\mathrm{C}$ terminal, including the site responsible for interacting with the calcium-channel $\alpha 1$ poreforming subunit. As a result, the mutation is predicted to cause a loss of $\beta 4$ function. Electrophysiologically, the $\beta 4$ subunit is thought to act as a modulator that increases calcium conductance via the main pore. Lethargic mutants display reduced calcium conductance via P/Q-type calcium channels (Lin et al., 1999) as well as an apparent compensatory increase in the expression of other $\beta$ subunits (McEnery et al., 1998; Lin et al., 1999).

The $\beta 4$ subunit is normally expressed at high levels in the cerebellum, with lower levels in the cortex, hippocampus, thalamus, and other regions (Tanaka et al., 1995; Davanzo et al., 1998). At the neuropathological level, no overt abnormalities have been identified in the brain, spinal cord, nerve, or muscle (Dung and Swigart, 1972). The brain regions responsible for paroxysmal dyskinesias and the pathophysiological mechanisms by which changes in calcium conductances generate paroxysmal dyskinesias in lethargic mice remain to be determined.

\section{Relationship with epilepsy}

Lethargic mice display frequent polyspike discharges in association with brief staring spells. As a result, these mutants have been studied extensively as models for absence epilepsy (Hosford et al., 1995a,b; Aizawa et al., 1997; Hosford and Wang, 1997). However, the polyspike discharges can be readily dissociated from the paroxysmal dyskinesias (Fig. 7), indicating that these two phenomena represent distinct pleiotropic consequences of the same genetic defect.

The paroxysmal movement disorders are not currently considered to be forms of epilepsy, primarily because of the absence of detectable EEG correlates. However, several similarities between the two disorders have been recognized repeatedly. These similarities include the frequent presence of a prodromal aura, an episodic temporal profile, and a beneficial response to anticonvulsants. In addition, the simultaneous occurrence of paroxysmal movement disorders and epilepsy has been reported for several different families (Beaumanoir et al., 1996; Szepetowski et al., 1997; Guerri et al., 1999; Zuberi et al., 1999; Guerrini, 2001). The association of paroxysmal movement disorders and epilepsy also appears to occur in mutant mice; paroxysmal dyskinesias and polyspike discharges appear to occur in lethargic mice (Figs. 3, 7), tottering mice (Kaplan et al., 1979; Noebels and Sidman, 1979; Fureman et al., 2002), and ducky mice (Barclay et al., 2001).

The lack of EEG abnormalities in the paroxysmal dyskinesias may reflect the fact that traditional EEG methods measure rhythmic discharges that involve the cerebral cortex and are relatively insensitive to rhythmic discharges that involve strictly subcortical sites such as the basal ganglia or cerebellum. The detection of such discharges from subcortical sites might encourage the reclassification of the paroxysmal dyskinesias as a form of subcortical epilepsy. Alternatively, many of the similarities between paroxysmal dyskinesias and epilepsy may reflect a shared underlying mechanism, dysfunction of ion channels (Guerrini, 2001).

\section{REFERENCES}

Aizawa M, Ito Y, Fukuda H (1997) Pharmacologic profiles of generalized absence seizures in lethargic, stargazer, and $\gamma$-hydroxybutyratetreated mice. Neurosci Res 29:17-25.

Barchi RL (1998) Ion channel mutations affecting muscle and brain. Curr Opin Neurol 11:461-468.

Barclay J, Balaguero N, Mione M, Ackerman SL, Letts VA, Brodbeck J, Canti C, Meir A, Page KM, Kusumi K, Perez-Reyes E, Lander ES, Frankel WN, Gardiner RM, Dolphin AC, Rees M (2001) Ducky mouse phenotype of epilepsy and ataxia is associated with mutations in the Cacna2d2 gene and decreased calcium channel current in cerebellar Purkinje cells. J Neurosci 21:6095-6104.

Beaumanoir A, Mira L, Van Lierde A (1996) Epilepsy or kinesigenic choreoathetosis? Brain Dev 18:139-141.

Bhatia KP, Griggs RC, Ptacek LJ (2000) Episodic movement disorders as channelopathies. Mov Disord 15:429-433.

Burgess DL, Jones JM, Meisler MH, Noebels JL (1997) Mutation of the $\mathrm{Ca}^{2+}$ channel $\beta$ subunit gene $C \operatorname{chb} 4$ is associated with ataxia and seizures in the lethargic (lh) mouse. Cell 88:385-392.

Campbell DB, Hess EJ (1999) L-type calcium channels contribute to the tottering mouse dystonic episodes. Mol Pharmacol 55:23-31.

Davanzo PA, Belin TR, Widawski MH, King BH (1998) Paroxetine treatment of aggression and self-injury in persons with mental retardation. Am J Ment Retard 102:427-437.

Demirkiran M, Jankovic J (1995) Paroxysmal dyskinesias: clinical features and classification. Ann Neurol 38:571-579.

Dickie MM (1964) Lethargic $(l h)$. Mouse News Lett 30:31.

Doyle J, Ren X, Lennon G, Stubbs L (1997) Mutations in the Cacnl1a4 calcium channel gene are associated with seizures, cerebellar degeneration, and ataxia in tottering and leaner mutant mice. Mamm Genome 8:113-120.

Dung HC, Swigart RH (1971) Experimental studies of "lethargic" mutant mice. Tex Rep Biol Med 29:273-288.

Dung HC, Swigart RH (1972) Histo-pathologic observations of the nervous and lymphoid tissues of "lethargic" mutant mice. Tex Rep Biol Med 30:23-39.

Fahn S, Marsden CD (1994) The paroxysmal dyskinesias. In: Movement disorders 3 (Marsden CD, Fahn S, eds), pp 310-347. Oxford: Butterworth-Heinemann.

Fletcher CF, Lutz CM, O’Sullivan TN, Shaughnessy Jr JD, Hawkes R, Frankel WN Copeland NG, Jenkins NA (1996) Absence epilepsy in tottering mutant mice is associated with calcium channel defects. Cell 87:607-617.

Fletcher CF, Tottene A, Lennon VA, Wilson SM, Dubel SJ, Paylor R, Hosford DA, Tessarollo L, McEnery MW, Pietrobon D, Copeland NG Jenkins NA (2001) Dystonia and cerebellar atrophy in Cacna1a null mice lacking P/Q calcium channel activity. FASEB J 15:1288-1290.

Fureman BE, Jinnah HA, Hess EJ (2002) Triggers of paroxysmal dyskinesias in the calcium channel mouse mutant tottering. Pharmacol Biochem Behav 73:631-637.

Greenberg DA (1997) Calcium channels in neurological disease. Ann Neurol 42:275-282.

Guerri R, Bonanni P, Nardocci N, Parmeggiani L, Piccirilli M, De Fusco M, Aridon P, Ballabio A, Crozzo RCG (1999) Autosomal recessive rolandic epilepsy with paroxysmal exercise-induced dystonia and writer's cramp: delineation of the syndrome and gene mapping to chromosome 16p12-11.2. Ann Neurol 45:344-352.

Guerrini R (2001) Idiopathic epilepsy and paroxysmal dyskinesia. Epilepsia 42 [Suppl 3]:36-41.

Hosford DA, Wang Y (1997) Utility of the lethargic (lh/lh) mouse model of absence seizures in predicting the effects of lamotrigine, vigabatrin, tiagabine, gabapentin, and topiramate against human absence seizures. Epilepsia 38:408-414.

Hosford DA, Lin FH, Kraemer DL, Cao Z, Wang Y, Wilson JT (1995a) Neural network of structures in which $\mathrm{GABA}_{\mathrm{B}}$ receptors regulate absence seizures in the lethargic (lh/lh) mouse model. J Neurosci 15:7367-7376. 
Hosford DA, Wang Y, Liu CC, Snead OC (1995b) Characterization of the antiabsence effects of SCH 50911, a GABA-B receptor antagonist, in the lethargic mouse, $\gamma$-hydroxybutyrate, and pentylenetetrazole models. J Pharmacol Exp Ther 274:1399-1403.

Jen J (1999) Calcium channelopathies in the central nervous system. Curr Opin Neurobiol 9:274-280.

Jouvenceau A, Eunson LH, Spauschus A, Ramesh V, Zuberi SM, Kullmann DM, Hanna MG (2001) Human epilepsy associated with dysfunction of brain P/Q-type calcium channel. Lancet 358:801-807.

Jun K, Piedras-Renteria ES, Smith SM, Wheeler DB, Lee SB, Lee TG, Chin H, Adams ME, Scheller RH, Tsien RW, Shin HS (1999) Ablation of P/Q-type $\mathrm{Ca}^{2+}$ channel currents, altered synaptic transmission, and progressive ataxia in mice lacking the $\alpha 1 \mathrm{~A}$-subunit. Proc Natl Acad Sci USA 96:15245-15250.

Kaplan BJ, Seyfreid TN, Glaser GH (1979) Spontaneous polyspike discharges in an epileptic mutant mouse (tottering). Exp Neurol 66:577-586.

Kertesz A (1967) Paroxysmal kinesigenic choreoathetosis: an entity within the paroxysmal choreoathetosis syndrome: description of 10 cases, including 1 autopsied. Neurology 17:680-690.

Lance JW (1977) Familial paroxysmal dystonic choreoathetosis and its differentiation from related syndromes. Ann Neurol 2:285-293.

Letts VA, Felix R, Biddlecome GH, Arikkath J, Mahaffey CL, Valenzuela A, Bartlett FS, Mori Y, Campbell KP, Frankel WN (1998) The mouse stargazer gene encodes a neuronal $\mathrm{Ca}^{2+}$ channel $\gamma$ subunit. Nat Genet 19:340-347.

Lin FH, Sureyya B, Lutz CM, Wang Y, Hosford DA (1999) Decreased ${ }^{45} \mathrm{Ca}^{2+}$ uptake in $\mathrm{P} / \mathrm{Q}$-type calcium channels in homozygous lethargic $\left(\right.$ cacnb $\left.4^{\text {lh }}\right)$ mice is associated with increased $\beta 3$ and decreased $\beta 4$ calcium channel subunit mRNA expression. Brain Res Mol Brain Res 71:1-20.

McEnery MW, Copeland TD, Vance CL (1998) Altered expression and assembly of N-type calcium channel $\alpha_{1 \mathrm{~B}}$ and $\beta$ subunits in epileptic lethargic $(\mathrm{lh} / \mathrm{lh})$ mouse. J Biol Chem 273:21435-21438.

Mori Y, Wakamori M, Oda S, Fletcher CF, Sekiguchi N, Mori E, Cope- land NG, Jenkins NA, Matsushita K, Matsuyama Z, Imoto K (2000) Reduced voltage sensitivity of activation of P/Q-type $\mathrm{Ca}^{2+}$ channels is associated with the ataxic mouse mutant rolling Nagoya $\left(\operatorname{tg}^{\text {rol }}\right)$. J Neurosci 20:5654-5662.

Munchau A, Valente EM, Shahidi GA, Eunson LH, Hanna MG, Quinn NP, Schapira AHV, Wood NW, Bhatia KP (2000) A new family with paroxysmal exercise induced dystonia and migraine: a clinical and genetic study. J Neurol Neurosurg Psychiatry 68:609-614.

Noebels JL, Sidman RL (1979) Inherited epilepsy: spike-wave and focal motor seizures in the mutant mouse tottering. Science 204:1334-1336.

Sadamatsu M, Masui A, Sakai T, Kunugi H, Nanko S, Kato N (1999) Familial paroxysmal kinesigenic choreoathetosis: an electrophysiological and genotypic analysis. Epilepsia 40:942-949.

Sahgal A (1993) Behavioral neuroscience: a practical approach. New York: Oxford UP.

Szepetowski P, Rochette J, Berquin P, Piussan C, Lathrop GM, Monaco AP (1997) Familial infantile convulsions and paroxysmal choreoathetosis: a new neurological syndrome linked to the pericentromeric region of human chromosome 16. Am J Hum Genet 61:889-898.

Tanaka O, Sakagami H, Kondo H (1995) Localization of mRNAs of voltage-dependent $\mathrm{Ca}^{2+}$-channels: four subtypes of $\alpha 1$ - and $\beta$-subunits in developing and mature rat brain. Brain Res Mol Brain Res 30:1-16.

Todorova MT, Burwell TJ, Seyfried TN (1999) Environmental risk factors for multifactorial epilepsy in EL mice. Epilepsia 40:1697-1707.

Tournier-Lasserve E (1999) CACNA1A mutations: hemiplegic migraine, episodic ataxia, type 2, and others. Neurology 53:3-4.

Zuberi SM, Eunson LH, Spauschus A, De Silva R, Tolmie J, Wood NW, McWilliam RC, Stephenson JPB, Kullmann DM, Hanna MG (1999) A novel mutation in the human voltage-gated potassium channel gene (Kv1.1) associated with episodic ataxia type 1 and sometimes partial epilepsy. Brain 122:817-825.

Zwingmann T, Neumann PE, Noebels JL, Herrup K (2001) Rocker is a new variant of the voltage-dependent calcium channel gene cacnala. J Neurosci 21:1169-1178. 